microstructure, cellular automata, finite element method, spring algorithm

Lukasz MADEJ ${ }^{1 *}$, Mateusz SITKO ${ }^{1}$, Aleksander FULAR ${ }^{1}$, Rafal SARZYN ${ }^{1}$, Mariusz WERMINSKI ${ }^{1}$, Konrad PERZYNSKI $^{1}$

\title{
CAPTURING LOCAL MATERIAL HETEROGENEITIES IN NUMERICAL MODELLING OF MICROSTRUCTURE EVOLUTION
}

\begin{abstract}
The work focuses on developing the complex digital shadow of the metallic material microstructure that can predict its evolution during metal forming operations. Therefore, such a digital shadow has to consider all major physical mechanisms influencing the particular investigated phenomenon. The motivation for the work is directly related to the development of modern metallic materials, often of multiphase nature. Such microstructure types lead to local heterogeneities influencing material behaviour and eventually macroscopic properties of the final product. The concept of the digital material shadow, stages of the model development, and examples of practical applications to simulation of microstructure evolution are presented within the work. Capturing local heterogeneities that have a physical origin and eliminating numerical artefacts is particularly addressed. Obtained results demonstrate the capabilities of such a digital microstructure shadow approach in the numerical design of final product properties.
\end{abstract}

\section{INTRODUCTION}

Development of the economy based on knowledge is considered an imminent priority for dynamically developing countries that need to face various challenges associated with, e.g., environmental protection [1,2]. That is particularly important for the manufacturing sector that needs to adjust to new strict requirements and introduce advanced engineering materials and their processing operations to everyday production to maintain competitiveness and satisfy customers' demands. As a result, highly complex metallic $[3,4]$ and composite $[5,6]$ materials developed previously with a very narrow scope of application, e.g., for space exploration, are being adapted to everyday production. Often, only they can deliver the required performance within the range of environmental restrictions. They have to be introduced to airplanes, cars, or motorcycles to reduce their weight, fuel consumption, emissions etc., and at the same time still increase safety factors of final components [7]. But the trend is more global and affects all the industrial branches like chemical, electronics or even medicine.

\footnotetext{
${ }^{1}$ Faculty of Metals Engineering and Industrial Computer Science, AGH University of Science and Technology, Poland

*E-mail: 1madej@agh.edu.pl https://doi.org/10.36897/jme/143086
} 
For many years, novel materials were being designed and developed through costly and time-consuming experimental and laboratory research that often has investigative limitations [8]. At the same time, recent progress in computer systems and available computation power opened new perspectives for computer-aided engineering approaches [9]. That creates new and often unrecognized opportunities for materials science applications that complement and extend experimental investigations. Designing virtual/digital shadows [10] that accurately describe materials concerning their actual microstructure with grains, phases, defects, or even atoms is now a broad and multidisciplinary research area [11-14]. With such digital engineering tools, scientists can analyse all microstructure components that are often invisible during experimental observations of processing and exploitation conditions. However, when microscale features of complex multiphase microstructures [15] are numerically investigated, appropriate capturing of the local heterogeneities becomes critical. These local material interactions eventually result in specific macroscopic behaviours of the final product [16]. Therefore, very sophisticated numerical modelling tools often have to be used, but most are based on two-dimensional investigation [17, 18]. Unfortunately, when complex phase materials are investigated, 2D analysis can be misleading as the microstructure morphology in the third direction cannot be captured $[19,20]$. That is why three-dimensional models are required, but that significantly increases the complexity of the investigation.

Therefore, the development of such 3D digital microstructure shadow is the overall goal of this research. The current case study is based on the numerical investigation of local heterogeneities during the 3D deformation of polycrystalline steel and the identification of their influence on further microstructure evolution during heat treatment operation. The mesh-based, finite element (FE) software was selected to investigate the former aspect and the Cellular Automata (CA) method for evaluation of the latter stage. In this case study, a particular issue that has to be solved is a proper discretization of the three-dimensional computational domain with the finite elements to eliminate any unphysical mesh influence that may occur at this length scale and then can artificially affect further CA simulations.

The findings indicate that the application of such a three-dimensional digital shadow model of microstructure that is properly discretized for the numerical simulation of deformation can provide valuable information on local heterogeneities during material processing. At the same time, it was highlighted that such research requires a combination of various dedicated numerical techniques.

\section{DIGITAL MATERIAL REPRESENTATION}

The Digital Material Representation (DMR) concept is considered a digital shadow of the investigated microstructure that can accurately capture local phenomena occurring during thermo-mechanical treatment under production and exploitation conditions [21]. The main objective of the DMR is a digital reconstruction of the microstructure morphology with its features (grains, grain boundaries, phases, inclusions, defects etc.) represented explicitly to replicate metallographic observations. The generation of such a digital shadow model both in 2D and 3D space is based on three major steps (Fig. 1): 
1. Numerical reconstruction of the microstructure morphology.

2. Identification of local material properties at the level of single grains.

3. Incorporation of the digital microstructure features with its properties into numerical software based on, e.g., finite element approach.

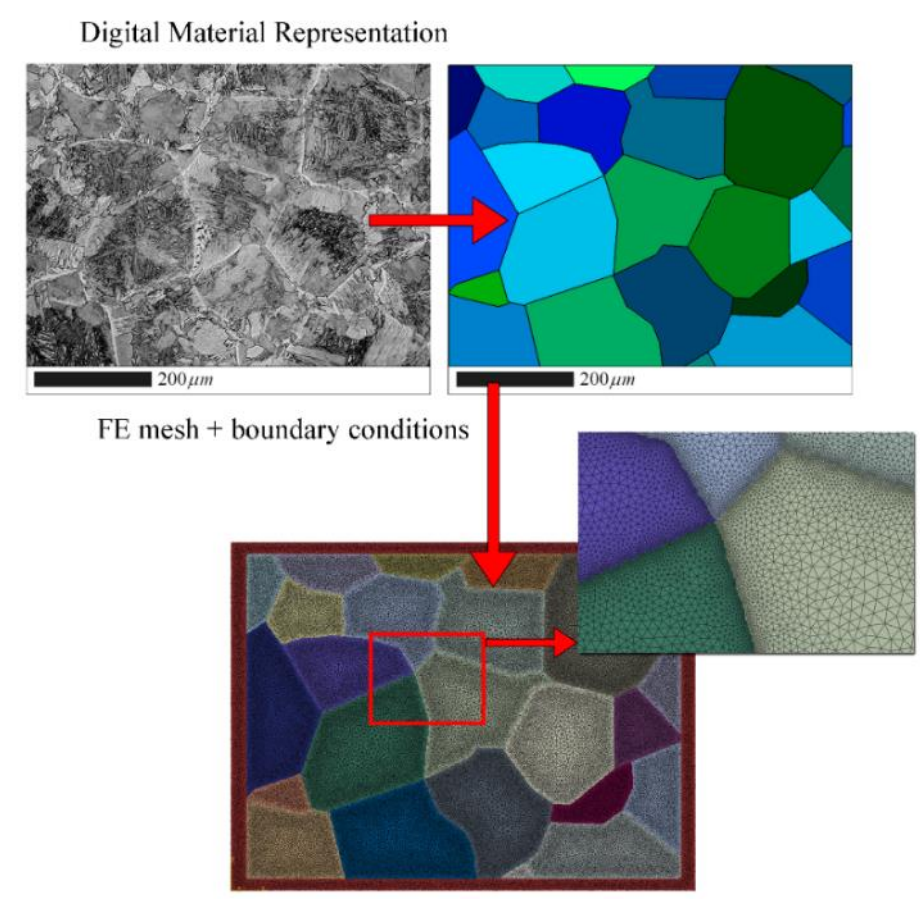

Fig. 1 Generation of the digital material representation model for subsequent calculations

The paper focuses on the third step as it directly deals with capturing local heterogeneities and eliminating unphysical artifacts that affect model description during subsequent calculations. Details on the first two steps related to the generation of 2D/3D digital morphologies and identifying local material properties can be found in earlier authors' work [22]. Therefore, only major information is summarized below for the clarity of the manuscript.

In the first step, the digital models with specific local features are most often created as exact replicas of microstructures, based on image processing of metallography data acquired from light (LM) or electron (EM) microscopy in two-dimensional space. However, as mentioned, such 2D representation is often insufficient. Therefore, three-dimensional data are required, which makes the experimental and numerical processing procedures more laborious. In the simplest case, a serial sectioning approach [23] can provide a series of 2D images from the subsequent depth of the material. Then 3D reconstruction algorithms have to be used to recreate complete volumetric information on microstructure morphology. The serial sectioning can be based on LM images allowing reconstruction of grain morphology, but also on, e.g., electron backscattered diffraction (EBSD) maps, that also provide information on crystallographic orientation along with its local variations. The concept of the EBSD based serial sectioning in application to two-phase ferritic-pearlitic steel is presented in Fig. 2. 


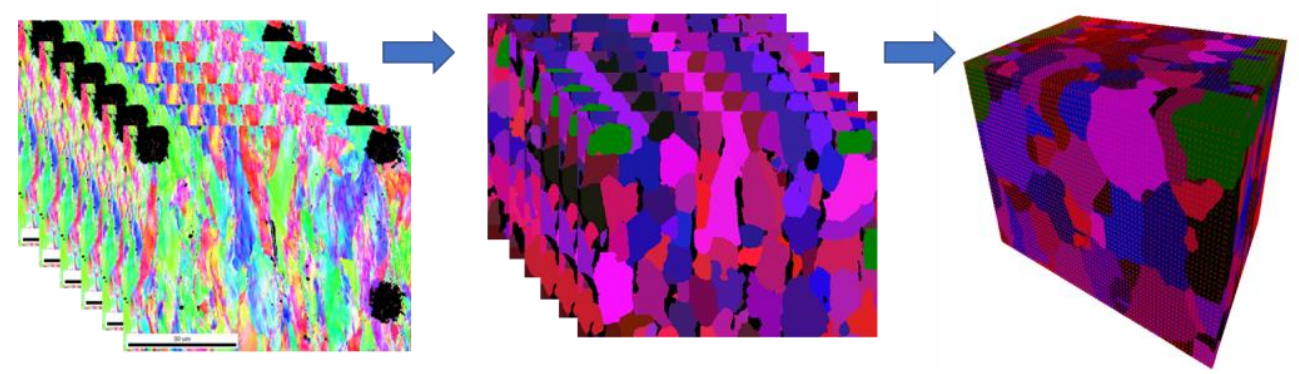

Fig. 2 Example of the EBSD based serial sectioning in application to ferritic-pearlitic steel

Unfortunately, serial sectioning is a destructive method, so there is no possibility to develop a digital model of the same area before and after deformation. In such a case, more sophisticated experimental procedures are required, e.g., computed tomography, X-ray diffraction contrast tomography, or near-field high-energy X-ray diffraction microscopy (nf-HEDM) [24]. These experimentally gathered data also require a set of data processing operations to provide meaningful information on microstructure morphology. In this case, these data are already in the volumetric form, and the methods are classified as nondestructive ones (Fig. 3). Access to appropriate equipment capable of acquiring a sufficient amount of data, not only about microstructure morphology but, e.g., on mentioned crystallographic orientation, is the major limitation in this case.

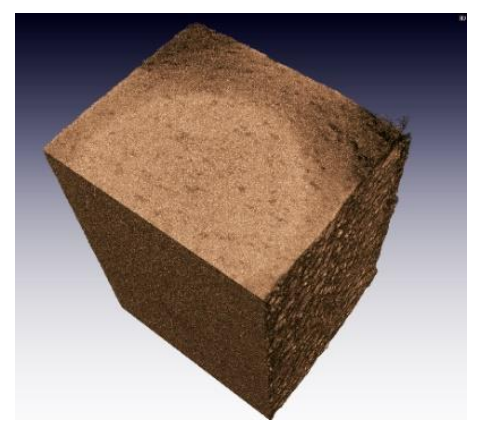

Fig. 3 Porous microstructure obtained from computed tomography analysis

Therefore, different numerical algorithms were proposed throughout the years to overcome these difficulties and generate synthetic digital microstructures, without the need for a large set of experimental data. The Voronoi Tessellation (VT) [25], Cellular Automata [26], Monte Caro (MC) [27] methods, sphere packing, or various hybrid approaches, which are a combination of the above-mentioned solutions, are being developed in this case, as seen in Fig. 4.

Customization of the hybrid solutions allows the generation of highly complex microstructure morphologies of metallic and non-metallic materials (complex multiphase steels, porous materials, metallic foams, granular structures, reinforced composites etc.), as seen in Fig. 5. Versatile capabilities in this area are provided by the DigiCore library developed in [21]. 
a)

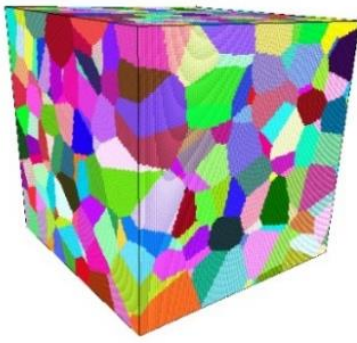

b)

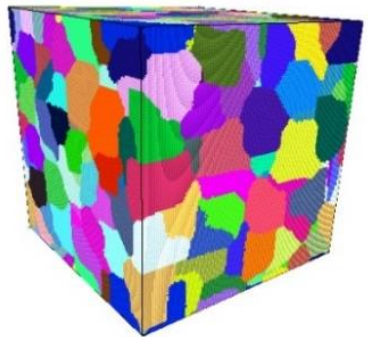

c)

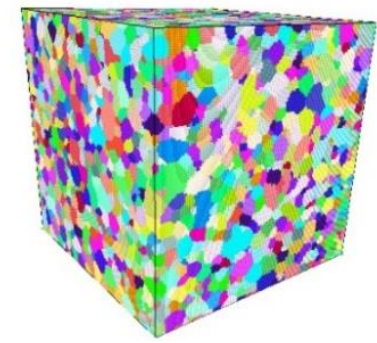

d)

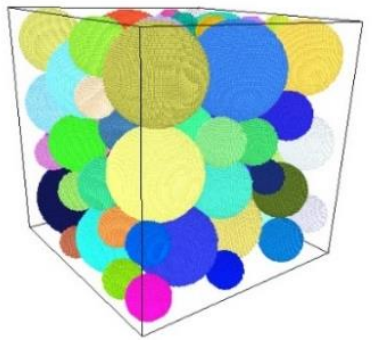

e)

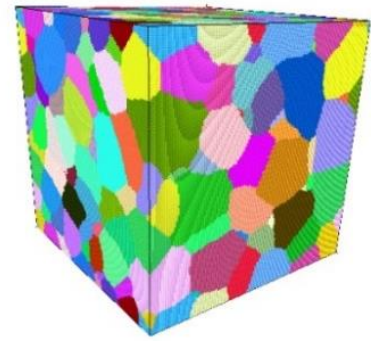

Fig. 4. Digital microstructure morphologies obtained with a) Voronoi Tessellation, b) Cellular Automata and c) Monte Carlo, d) sphere packing, and e) hybrid algorithm

a)

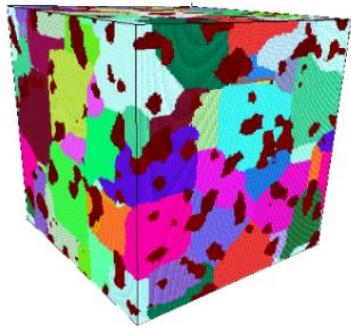

c)

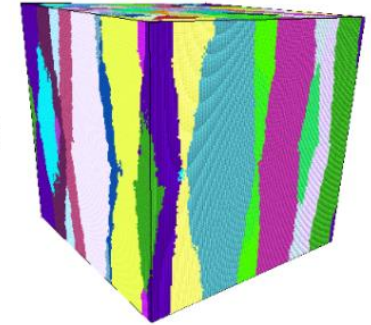

d)

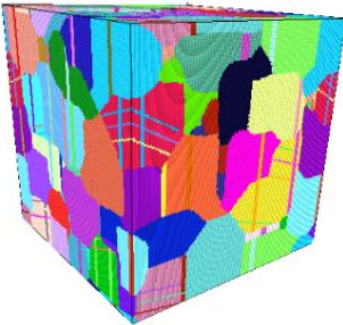

b)

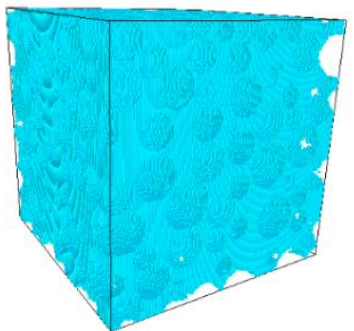

e)

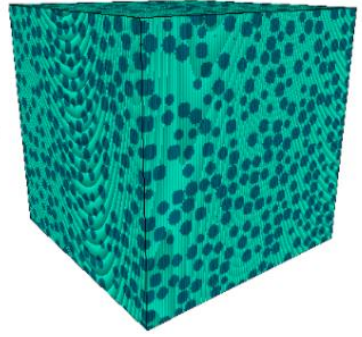

Fig. 5. Digital morphologies of complex metallic and non-metallic microstructures: a) multiphase steels, b) porous materials, c) thin film, d) twin boundaries, e) reinforced composites

In the second step of DMR generation, appropriate material properties are assigned to particular microstructural components. Local evaluation of material properties at the level of particular grains is again not a trivial task. The micro/nano mechanical testing equipment has to meet very high-quality standards to identify differences in properties associated with, e.g., different crystallographic orientations. Advanced specimen fixture systems, actuation systems, or load cells have to be used to deliver reliable and repetitive results. The most commonly used technique is based on the nano-indentation approach, but other solutions like micropillar compression, micro tensile, micro bending, or even micro torsion tests are also available [28]. Of course, all these tests have different levels of complexity/accuracy what affects their execution times and costs. 
Nevertheless, both steps mentioned above provide a detailed representation of local heterogeneities associated with microstructure morphology as well as properties. Finally, in the third step, appropriate space discretization algorithms have to be used in order to use such input data during subsequent finite element simulations of material behaviours under loading conditions. Commercial finite element codes do not provide sufficient mesh generation solutions for this particular application. Therefore, the development and implementation of specific algorithms are critical for the accuracy of further calculations.

\section{FINITE ELEMENT DISCRETIZATION CONFORMING LOCAL MICROSTRUCTURAL FEATURES}

The developed mesh generation algorithm is based on information from the digital microstructure representation model. In particular, the position of the grain boundary or other microstructure feature boundary is considered. The significant advantage of the proposed algorithm is the capability to create conforming FE meshes that are refined along boundaries of subsequent microstructure elements. That way, high accuracy of the numerical analysis is obtained in the regions with significant solution gradients while the computational time remains at the acceptable time. The finite element meshes for digital microstructures with non-periodic as well as periodic boundary conditions can be created during a set of subsequent steps:

- Parsing the input data from the digital microstructure model.

- Generating inner nodes along the microstructural features.

- Generating Delaunay conforming triangulation.

- Improving the discretization level.

- Assigning generated elements to particular microstructural features.

- Generating output data for further FE calculations.

As presented, the first important stage is the identification of boundary nodes within the input data structure. The boundary nodes separate subsequent microstructural features, e.g., grains, phase boundaries, inclusions etc. Explicit representation of such boundaries is the basis for the generation of good quality conforming meshes. Therefore, the developed approach takes an image of microstructure obtained experimentally or numerically, in the form of a bitmap in 2D or text file (.txt or .vtk) in the 3D space, as input data. In this case, it is essential to ensure during data processing operations that each colour in the input file corresponds to a unique microstructural feature (Fig. 6).

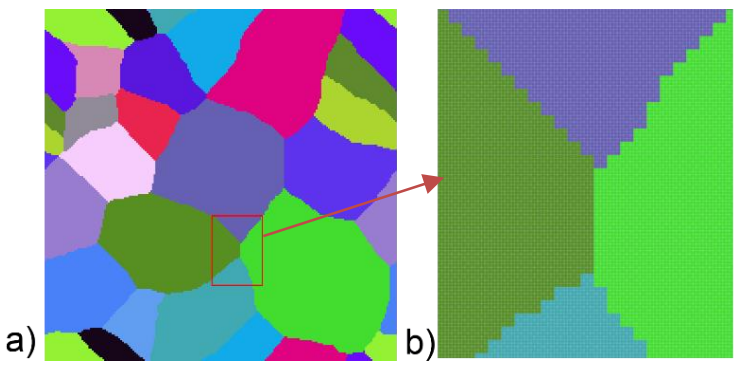

Fig. 6. Input data in the 2D format of the bmp file 
Therefore, the image cannot be smoothed by any edge smoothing algorithm, as that would generate many artifacts between subsequent features. This is often related to exporting the image into file format with lossy compression, as shown in Fig. 7.

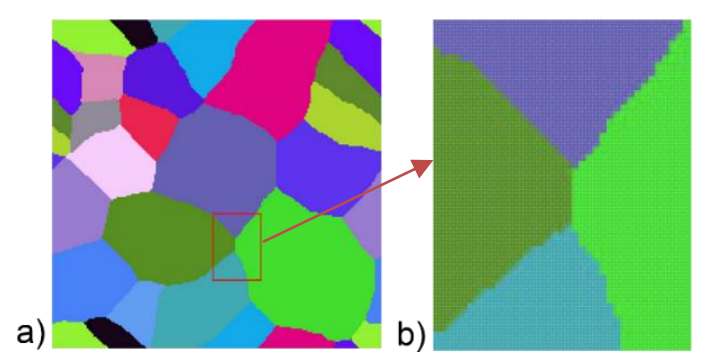

Fig. 7. Input data in the $2 \mathrm{D}$ format of the jpg file

A similar assumption is made in the 3D case, where each voxel of the input data is described by a unique id number assigning it to a particular feature, as seen in Fig. 8 .

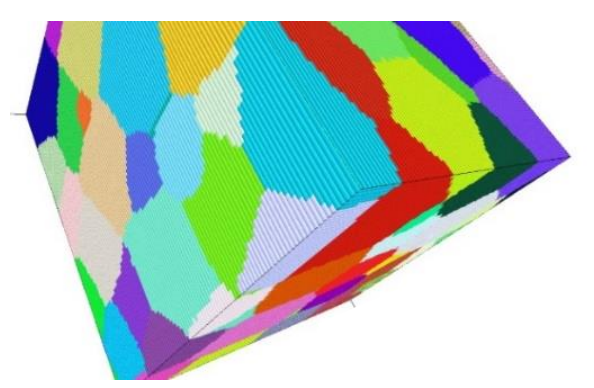

Fig. 8. Input data in the 3D space

During an import stage of the input file, an algorithm explicitly identifies the boundary between each set of features. Each pixel of the input image is investigated during this stage, with the Manhattan distance equal to one, commonly referred to as the Von Neumann neighborhood. When any neighbor of the investigated pixel is of different colour with respect to the analysed one, it is added to a list of boundary cells. The modified Von Neuman neighborhood is often used during the calculations (Fig. 9) to ensure that the thickness of the generated feature boundary is of single-voxel size. The same concept is applied in 3D cases. As a result, a clearly visible boundary between subsequent features is obtained for further processing, as seen in Fig. 10.
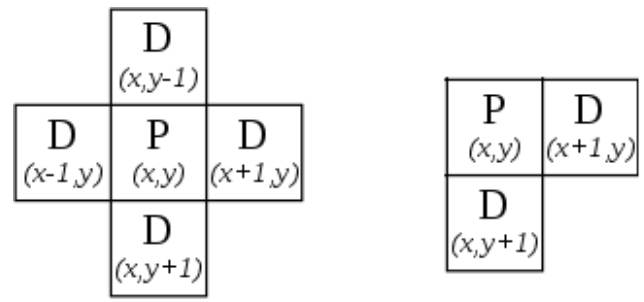

Fig. 9. Type of neighborhood in 2D case: a) classical Von Neumann, b) modified Von Neumann 
a)

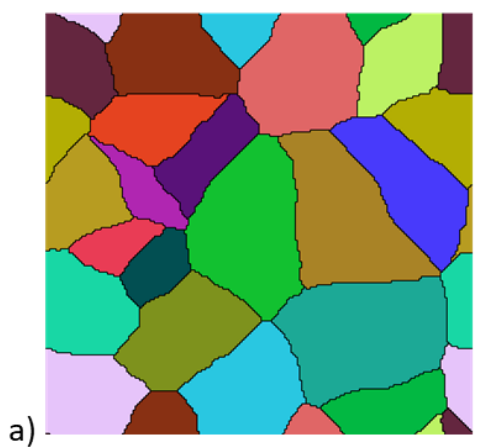

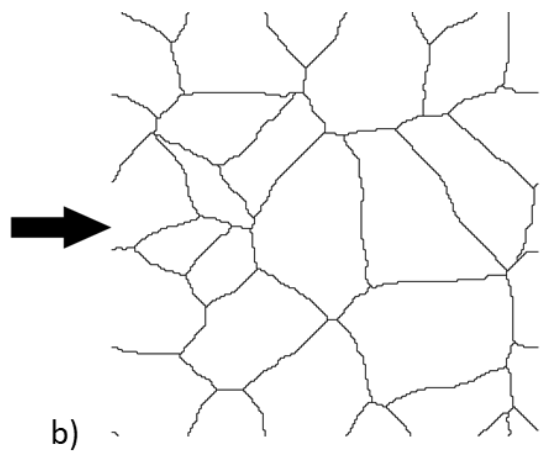

Fig. 10. Example of input data pre-processing operation: a) microstructure with coloured grains and grain boundaries, b) identified grain boundaries

The control parameter responsible for spreading out the node-set was also defined to minimize the number of nodes along the grain boundaries. The parameter can be from the range [0.1-1]. The value of, e.g., 0.5 will roughly result in a $50 \%$ reduction in the density of nodes along the grain boundaries.

In the case of generating mesh with periodic boundary conditions, additional modifications to the computational domain edges in $2 \mathrm{D}$ or faces in 3D were designed. The nodes generated along the edges/planes are mirror-copied to the corresponding edges/planes, as seen in Fig. 11.
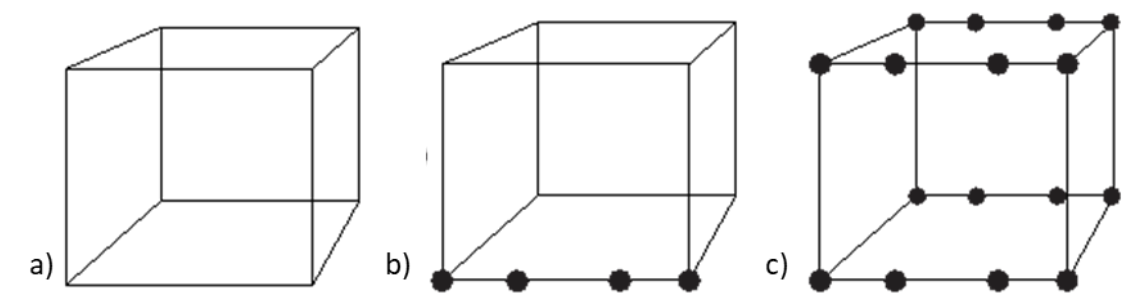

Fig. 11. Example of introducing periodic boundary nodes in the 3D computational domain

Then, the generation of nodes within the interior of investigated microstructural features is the second step of the algorithm. The distribution of new nodes is strictly controlled to obtain the refined size of finite elements in the area of feature boundaries and coarser element size in the interior of those features. The developed seeding algorithm assumes the generation of new inner nodes along the circumference of circles with a predefined radius. The concept of the algorithm is based on several steps:

- for the boundary nodes:

1. Generate a circle with a radius $r_{0}$ around subsequent points representing feature boundaries. The $r_{0}$ radius is calculated by multiplying the distance to the closest boundary node by a coefficient $\alpha \in(0,1)$, e.g., 0.4 . The smaller the $\alpha$, the finer mesh will be obtained.

2. For a particular boundary node, generate 16 equally distributed new nodes $N_{i}^{0}$ $(i \in[1,16])$ along the circumference of its $r_{0}$ circle. 
3. For each new node, $N_{i}^{0}$ generate a circle with radius $r_{j}$; if there are other nodes within the radius, eliminate this $N_{i}^{0}$ node. The $r_{j}$ radius is calculated by multiplying the distance to the closest node by a coefficient $\beta>1$, e.g., 1.2.

- for the new nodes:

4. For a particular remaining node $N_{i}^{k}$ ( $k$-subsequent iterations of the algorithm), generate 16 equally distributed new nodes $N_{i}^{k+1}$ along the circumference of its circle.

5. For each new node, $N_{i}^{k+1}$ generate a circle with radius $r_{j}$; if there are other nodes within the circle, eliminate this $N_{i}^{k+1}$ node. The $r_{j}$ radius is calculated by multiplying the distance to the closest node by a coefficient $\beta>1$.

6. Go back to step 4 for another $k$ iteration.

The following steps of the algorithm are summarized in Fig. 12.
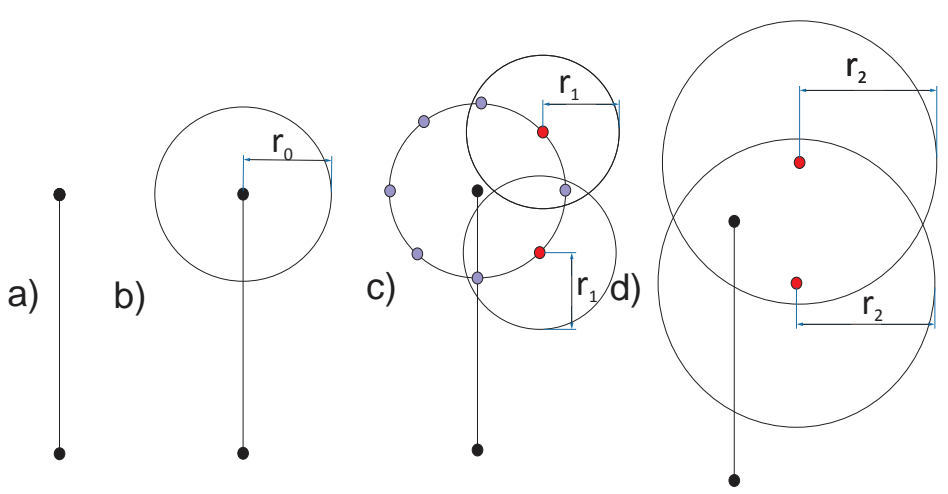

Fig.12. a) Boundary nodes, b) step 1, c) step 2, d) steps 4-6

In the 3D computational domain, a set of multiple rings additionally rotated along each axis is generated instead of drawing a single circle per node. Then a set of 16 new nodes along the circumference of each ring is investigated.

The circle-based generation of nodes provides relatively uniform node distribution in the computational domain, which increases the quality of further triangulation steps. Additionally, the KDTree [29] algorithm was implemented to identify the closest neighbors of the investigated node. The KDTree is well known for fast spatial searching of defined data structures. However, it does not deal well with node insertion, resulting in changes in the predefined data structure. Thus, to deal with the KDTree being gradually degenerated from the insertion of new nodes, a complete reinitialization of the tree data structure is performed after reaching a specific amount of newly added points, e.g., 750.

Finally, based on the generated nodal points, the Delaunay triangulation with the Bowyer-Watson (B-W) algorithm [30] was implemented to generate conforming meshes. The Bowyer-Watson algorithm allows discretization on the basis of available input nodes. The approach is based on an iterative adding subsequent nodes to the generated mesh what results in reconstruction of the local area of the mesh associated with the added vertex. These properties make this method an efficient tool for introducing changes in the density of the elements at every stage of the discretization process. 
a)

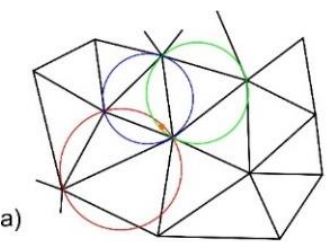

b)

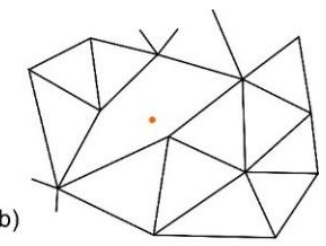

c)

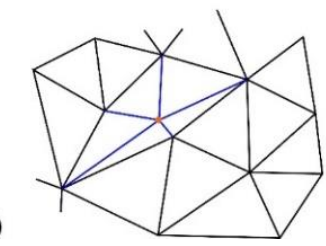

Fig. 13. Main steps of the B-W algorithm: a) insertion of the new node into the mesh and identification of all triangles that enclose new node in their circumcircles, b) elimination of identified triangles, c) re-triangulation with respect to the new point

The standard triangulation algorithm starts by forming the super triangle or combination of two super triangles (rectangle) enclosing all the investigated nodes. Then, incrementally, a process of inserting previously generated nodes into the mesh is performed. After every insertion, all triangles whose circumcircles contain the new point area are deleted, leaving a polygonal hole, which is then re-triangulated using the new point (Fig. 13).

The Delaunay triangulation is created when all the investigated points from the boundary and inner grain areas are inserted. As an alternative to the B-W implementation, a set of triangulation functions from the CGAL [31] library can also be used. The CGAL is a mature library, which provides means to generate both 2D and 3D triangulations based on a given set of points.

Quadrilateral elements can also be created within the developed approach based on the performed triangulation. First, generated triangles are merged with each possible neighbor to evaluate the best pair. The following equation is used as a criterion in the case:

$$
\Theta=1-\sqrt{2} \frac{r}{R}
$$

where: $R$ - maximum radius of the circle containing three nodes, $r$-minimum radius of the circle tangent to the three edges (Fig. 14.).

a)

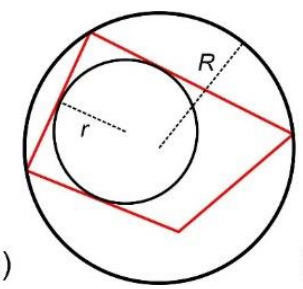

b)
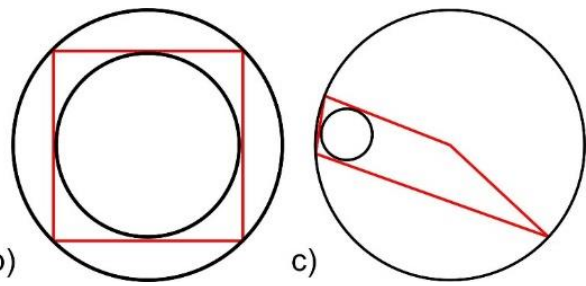

c)

Fig. 14. Illustration of the triangle merge criterion

As seen in Fig. 14, the closer $\Theta$ is to 0 , the closer the tetrahedral element is to a square. When the acceptable triangle pairs are created, a new node is inserted to each center of gravity of the rectangle and remaining unpaired triangles. Finally, those new points are connected to the centers of neighboring edges, resulting in good quality quadrilaterals (Fig. 15). 
a)

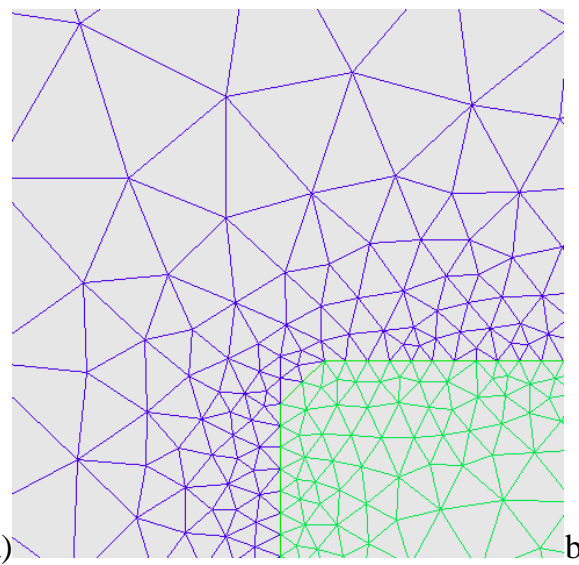

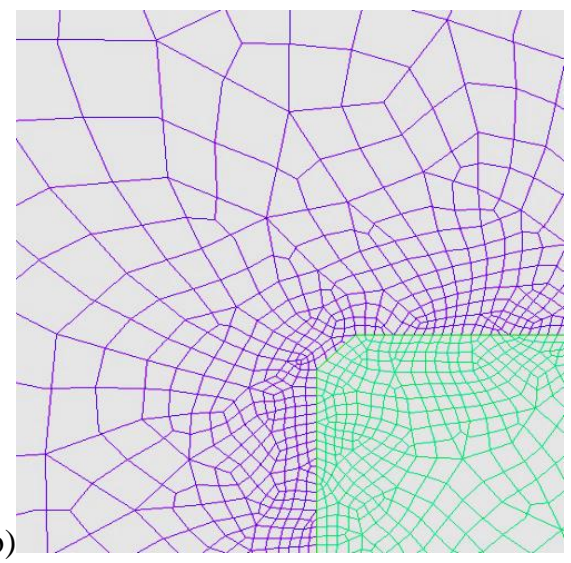

Fig. 15. Examples of a) initial triangular and b) final rectangle meshes

The crucial aspect from the mesh quality point of view to avoid unphysical heterogeneities during deformation simulations is the additional optimization step after initial triangulation. For that reason, the Laplace smoothing [31] and edge/face flipping algorithms are used. The basis of the Laplace smoothing assumes that each mesh node is shifted to a new position based on the position of the neighbors, as seen in Fig. 16.

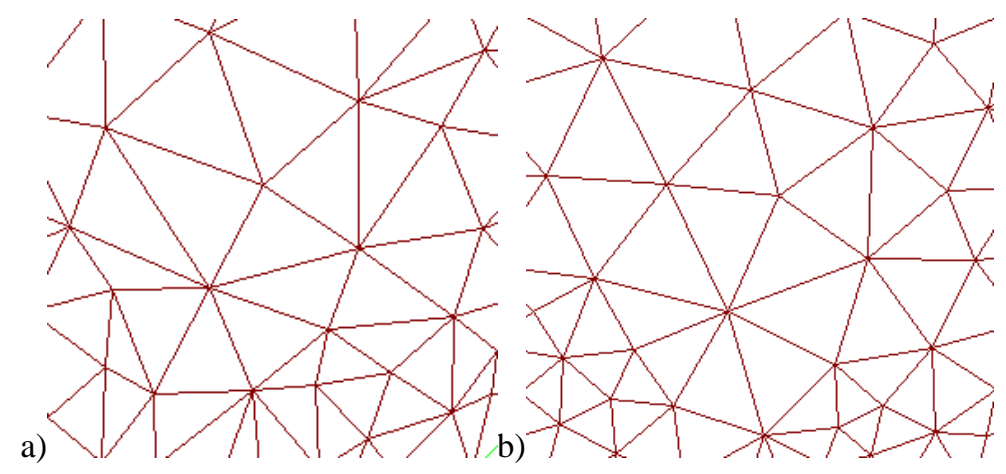

Fig. 16. Illustration of a) initial and b) the Laplace smoothed mesh

The edge/face flipping is another topological transformation implemented to remove elements from generated mesh and replace them with a different set of elements occupying the same space, as seen in Fig. 17. Performed flipping is a local operation where only a few elements are affected, e.g., 2 or 3. Usually, a combination of the two algorithms is sufficient to provide good quality meshes.
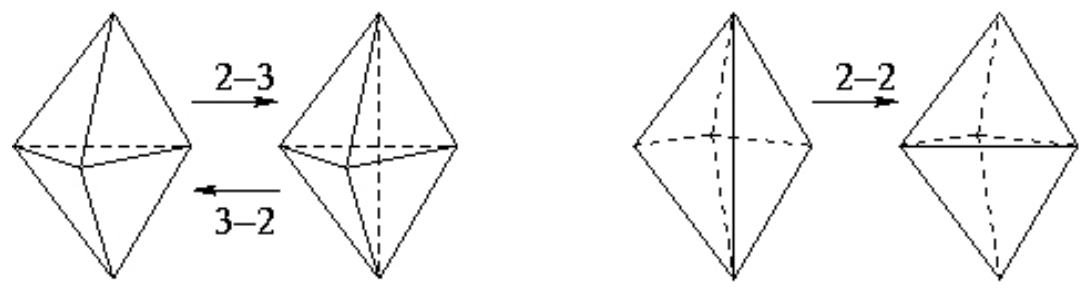

Fig.17. Presentation of topological face flipping concept in 3D case 
After the smoothing algorithm, the mesh may still contain degenerated elements that are elongated in one direction. In this case, a series of additional nodal points are added to the investigated space, and the mesh generation algorithm is performed again.

After the triangulation step, the generated elements are assigned to particular microstructural features based on information from the original input file in 2D or 3D. The assignment step can greatly impact simulation results, especially affecting the generation of local artificial heterogeneities unrelated to the microstructure morphology. The goal is to have the surface between the features as smooth as possible to reliably replicate the observed grain shape. Otherwise, artificial strain localization can occur in the solution what affects further microstructure evolution simulations. The developed algorithm is based on two stages. The first assigns the center mass of each element to the particular feature represented in the input data file by the discrete format. The second is related to smoothing the assigned elements located along the feature boundaries. Smoothing is realized in an iterative manner. In this case, multiple passes of reassigning of elements between features are performed to increase smoothness. In each pass, the algorithm identifies the neighbors of the investigated finite element that have the shared edge. Then, the number of neighboring finite elements that belong to the same grain is calculated. Finally, the investigated finite element is reassigned based on the majority of the neighbors belonging to the same grain, as presented in Fig. 18.

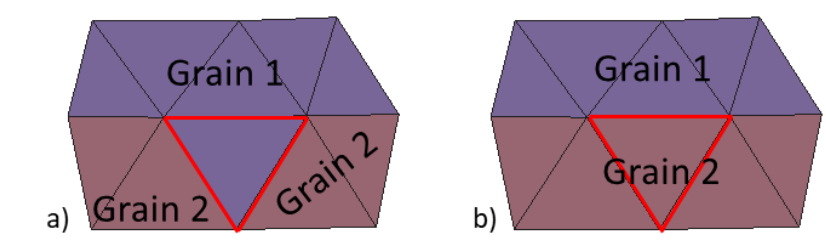

Fig. 18. Concept of the element reassignment algorithm

As mentioned, without this step, the element from Figa would lead to, e.g., strain or stress concentration and eventually to, e.g., fracture, which would not be related to the microstructure itself but to inappropriate mesh. Unfortunately, the approach does not provide satisfactory results in the 3D case; therefore, more advanced solutions were implemented in this case.

The 3D approach is based on the analogy to the behavior of springs [32]. It introduces a network of springs connecting all nodes in the mesh, as shown in Fig. 19. The variation of this method is the ball-vertex method, which improves the smoothing results thanks to the use of additional perpendicular linear springs (Fig. 20).

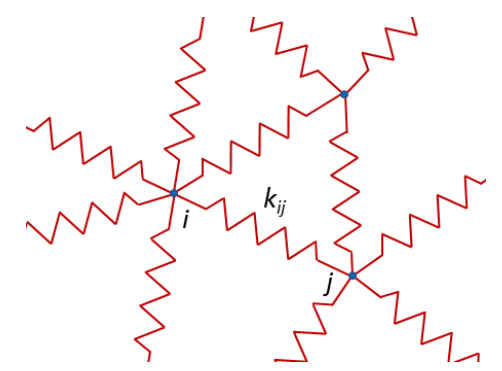

Fig. 19. System of springs in the 2D space [32] 


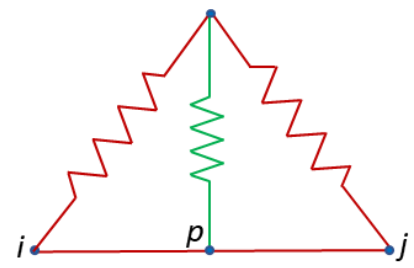

Fig. 20. Concept of the ball-vertex method in the 2D finite element [32]

After replacing the edges in the mesh with artificial springs, their stiffness, which is inversely proportional to the length, is calculated. By examining the $i$ - $j$ edge from Fig. 19, it is possible to calculate the force at the vertex $i$ exerted by the vertex $j$ :

$$
f_{i j}=k_{i j}\left(u_{j}-u_{i}\right) n_{i j} n_{i j}=-f_{j i}
$$

where: $k$ - the spring stiffness, $n_{i j}$ - the single-line vector from node $i$ to $j$. In this approach, it is important to check that the sum of forces associated with the investigated node is equal to 0 .

Finally, a good quality mesh is created, and elements are correctly assigned to particular features. The higher-order elements can also be formed by introducing additional nodes in the centers of edges in 2D and 3D, respectively, as seen in Fig. 21.

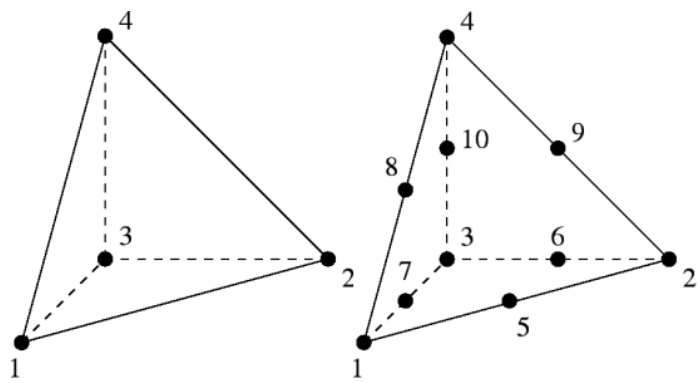

Fig. 21. Example of a) C3D4 and b) C3D10 elements

An illustration of the limitations of the classical Laplace smoothing algorithm is presented in Fig. 22b, while improvement obtained by the spring algorithm is shown in Fig. 22c.

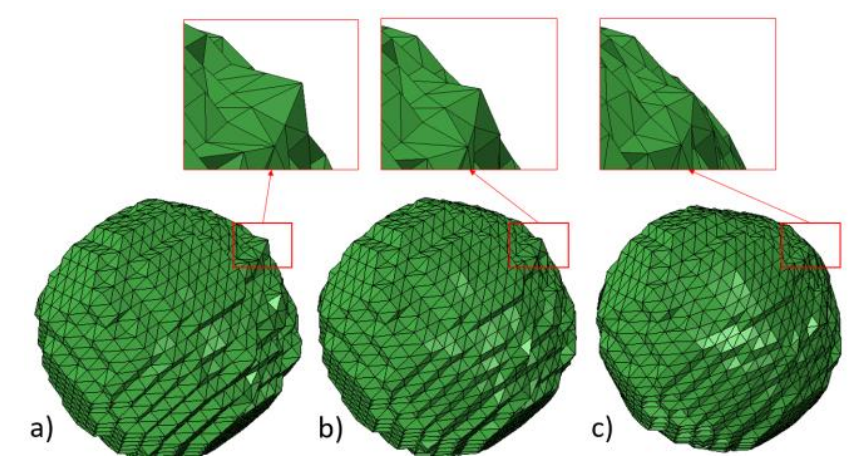

Fig. 22. Discretization of the sphere in the uniform matrix, a) after triangulation, b) after Laplace smoothing, c) after spring algorithm 
Finally, the last step of the digital shadow generation for subsequent finite element simulations is related to exporting the obtained mesh to the output file compatible with one of the available FE software. In the present case study, the output format is taken from the commercial Abaqus software and complies with all required sections in the input format file.

\section{APPLICATION OF THE DIGITAL MICROSTRUCTURE SHADOW TO MATERIAL FORMING CASE STUDY}

A tensile test at room temperature followed by the heat treatment operation was selected to present the capabilities of the developed methodology in capturing local material heterogeneities. The initial digital microstructure morphology was generated with the cellular automata grain growth model within the 3D computational domain. The size of the CA space was selected as $180 \times 180 \times 180$ cells with a physical size of $1 \mu \mathrm{m}$, as seen in Fig. 23 . The developed mesh generation algorithm with the spring smoothing technique was used for discretization purposes. The material is a ferritic-pearlitic steel, and the flow stress curve was identified based on the nanoindentation experiment. Additionally, to take into account the influence of various crystallographic orientations on the grain response, different flow stress curves, diversified with the Gauss distribution function, were assigned to the model.

The digital model was deformed at room temperature during the uniaxial tensile test experiment with periodic boundary conditions. The commercial Abaqus software was used for the calculation, where the constitutive equation is based on the $\mathrm{J} 2$ plasticity. The outcome of the simulations in the form of deformed microstructure morphology and heterogeneous distribution of accumulated strain is shown in Fig. 24. As presented, the DMR model can predict local heterogeneities in the deformed microstructure, which are crucial for material behavior during the subsequent processing operation. In the investigated case study, the deformed sample was then subjected to heat treatment where static recrystallization restores the deformed microstructure.

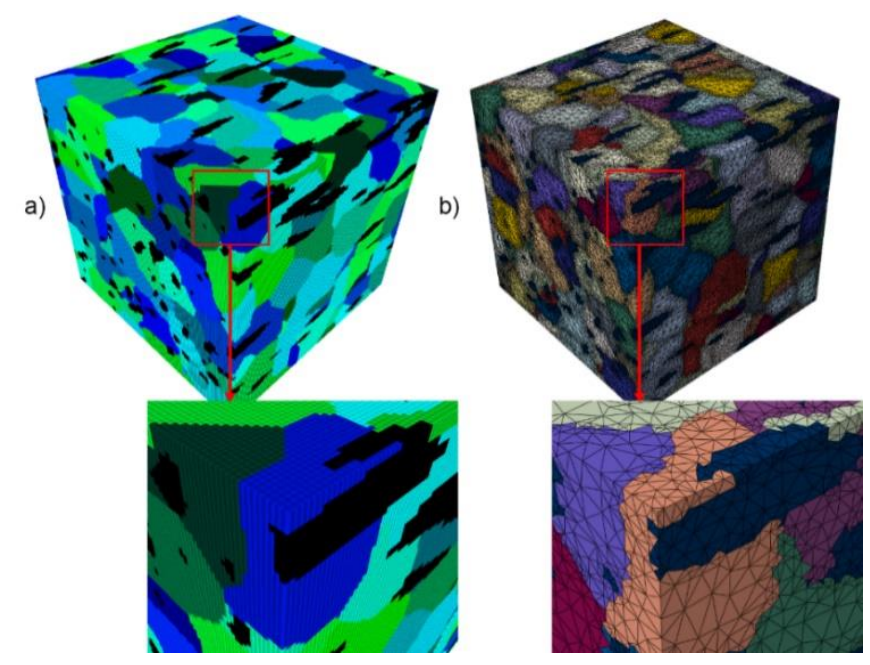

Fig. 23. Digital model of the $180 \times 180 \times 180 \mu \mathrm{m}$ microstructure after a) CA grain growth and b) finite element discretization operation 

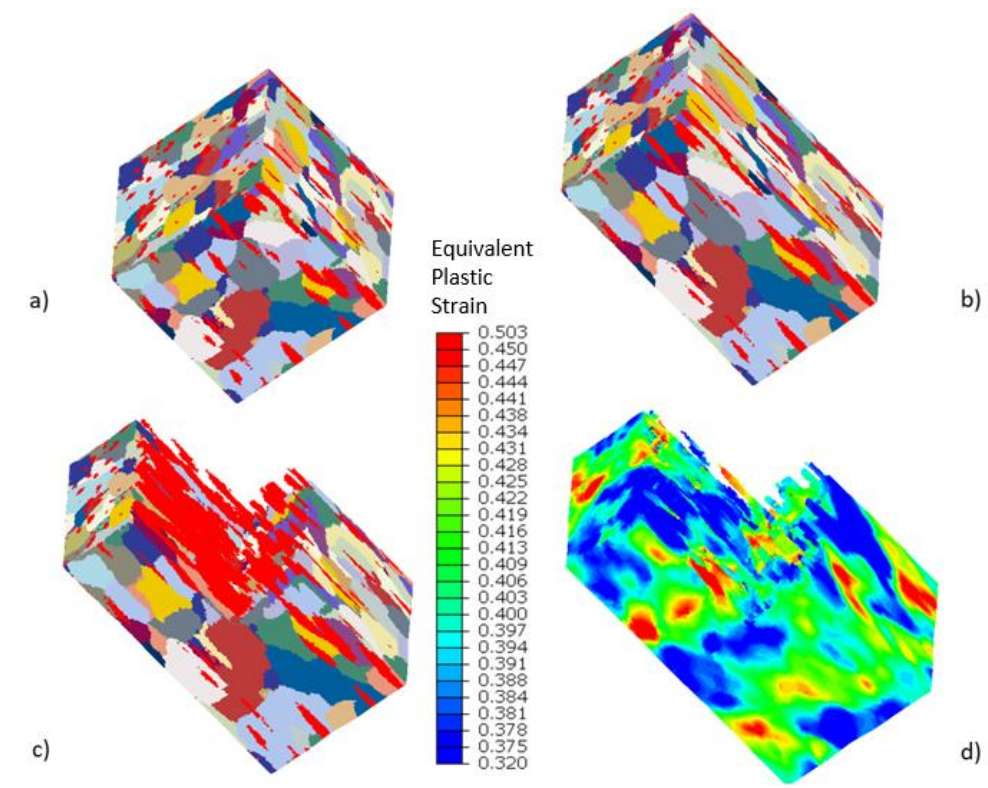

Fig. 24. Example of finite element results: a) undeformed and b, c) deformed microstructure morphology (with visible pearlite islands), d) heterogeneous distribution of accumulated strain

The static recrystallization is simulated with the developed CA model [33] that operates based on the data acquired from the deformed finite element mesh. The concept of the data flow between the CA and FE models that allows transferring deformed grain geometry along with the heterogeneous strain field is presented in Fig. 25.
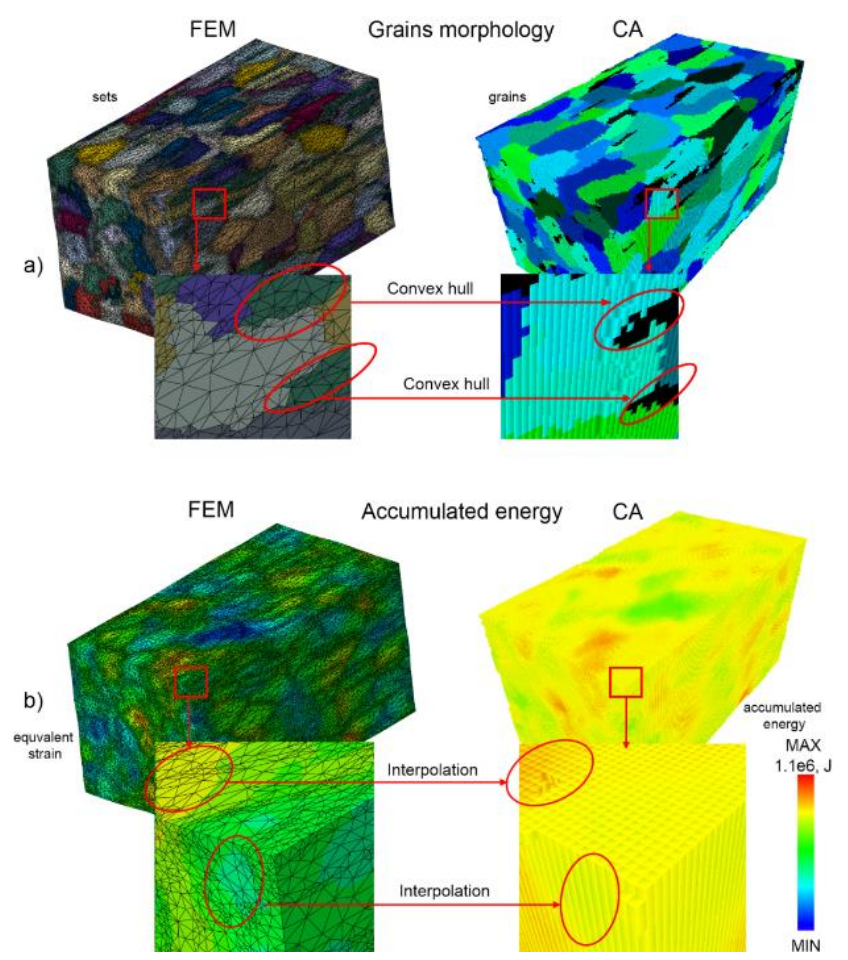

Fig. 25. Data transfer concept between FE and CA computational domains a) grains morphology, b) accumulated energy 

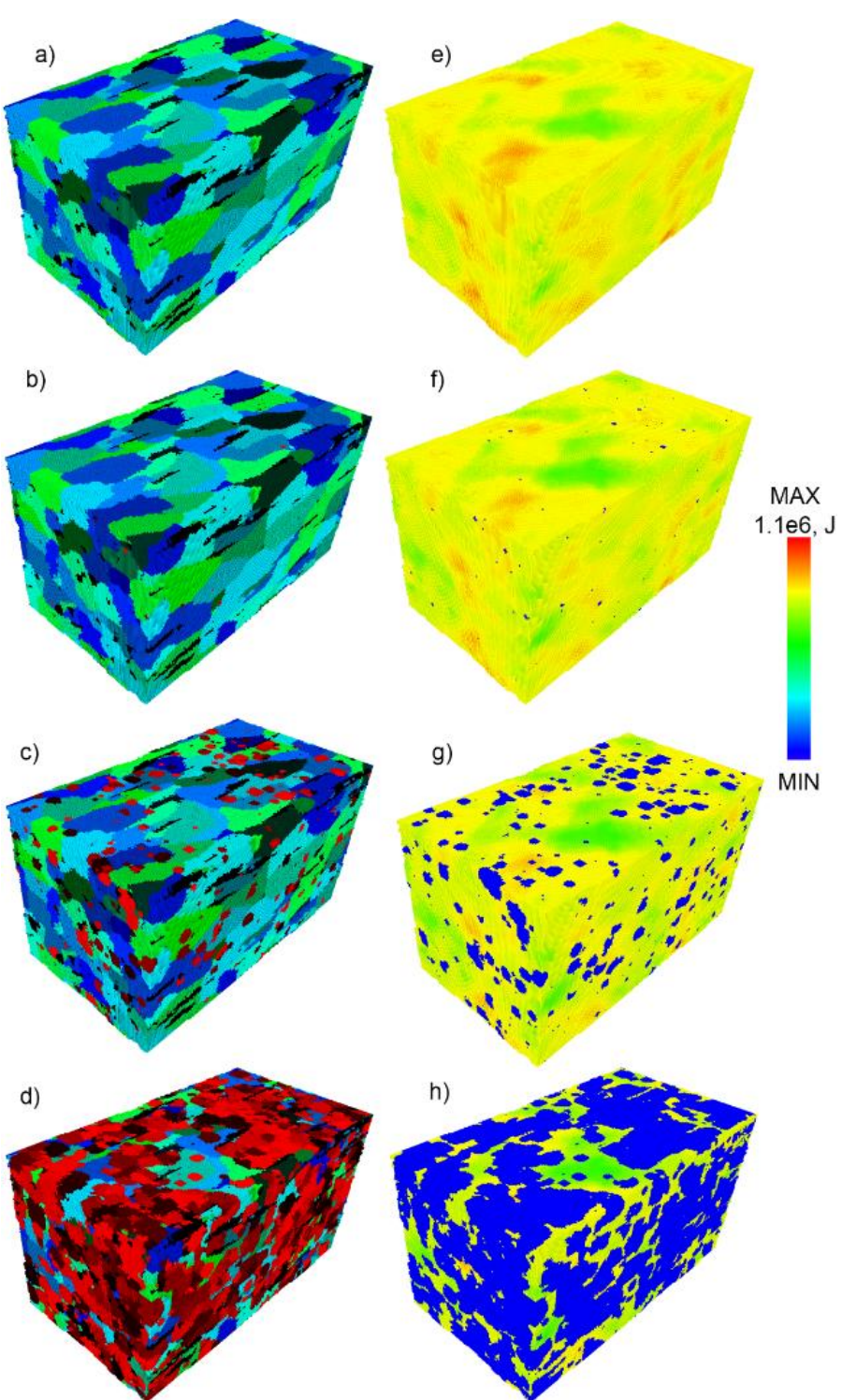

Fig. 26. Examples of CA simulation results: a) initial microstructure, b) - d) recrystallization progress, e) initial energy distribution, f) $-\mathrm{h}$ ) corresponding energy values during the simulation.

The CA model itself is based on the constitutive equation that relates the velocity of the grain growth with the mobility and the net pressure:

$$
v_{i}=M_{G} P \text {, }
$$

where: $M_{G}-$ grain boundary mobility, $P$ - net pressure on the grain boundary.

The net pressure, mostly related to the accumulated deformation energy value, is considered as the main driving force controlling the grain nucleation and growth during SRX. The stored energy field was obtained directly from presented DMR simulations. Details of the CA model can be found in [34], while examples of obtained results presenting static recrystallization progress within the deformed microstructure are shown in Fig. 26 and Fig. 27. 


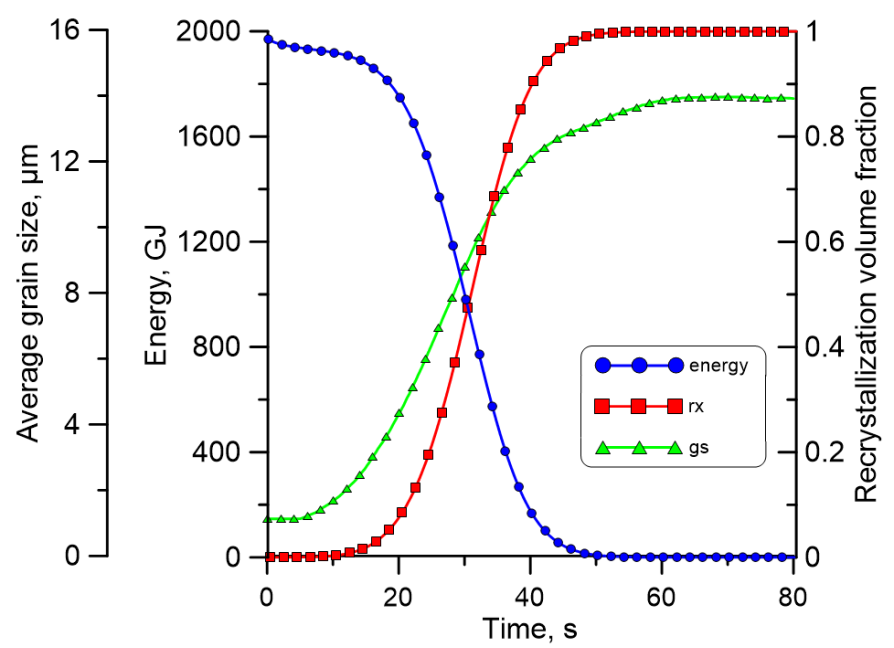

Fig. 27. Quantitative characterization of microstructure evolution during the CA simulation of static recrystallization, including the evolution of accumulated energy (energy), average grain size ( $g s)$, and recrystallized volume fraction ( $r x$ )

As can be observed in Fig. 26 the nucleation process initiates in the high-energy regions, and the grains grow into the surrounding deformed matrix. The SRX kinetics is accurately captured, with fast grain growth at the beginning and stagnation at the end of the process. This is directly attributed to the reduction of the stored energy due to SRX progress, as visible in Fig. 27. The fast growth of the average grain size is also limited at the end of the process. Combined characterization of microstructure evolution based on mean- and full-field data from Fig. 27 and Fig. 26, gives a complete overview of the grain evolution as well as their interactions with each other and pearlite islands. Such experimental investigation in the 3D space is still extremely rare in the literature due to the limitations of the experimental facilities. Therefore, the reliable digital shadow of the microstructure and computational material design concept is a very attractive alternative for the development of materials with tailored properties.

However, as pointed out in the paper, the elimination of numerical artefacts, affecting in an unphysical manner material behaviours, is critical.

\section{CONCLUSIONS}

The development of a complex digital shadow of the metallic material microstructure that can predict its evolution during subsequent stages of metal forming operations was presented within the work. The work is a part of ongoing research towards developing a digital shadow that considers all major physical mechanisms operating under particular processing conditions. As presented, the digital shadows, especially in 3D cases, significantly extend the capabilities of experimental investigation leading to an unprecedented level of understanding of the evolution of particular phenomena. However, the current research clearly pointed out that all numerical artifacts that affect the quality of numerical predictions have to be eliminated. In the case of the static recrystallization simulations, the most critical aspect is a reliable determination of the heterogeneities in the stored deformation energy. As discussed, 
these predictions can be significantly affected by the quality of initial microstructure morphology and FE discretization accuracy. The developed conforming mesh generation algorithm with smoothing options proved its robustness in the digital microstructure model simulations. The data transfer between finite element simulations of deformation and cellular automata microstructure evolution under heat treatment proved that the manufacturing chain can be simulated with such digital shadow.

The future work is directly related to the extension of the digital microstructure shadow capabilities towards the simulation of phenomena occurring under phase transformation conditions.

\section{ACKNOWLEDGMENT}

Financial assistance of the National Science Centre project No. 2017/27/B/ST8/00373 is acknowledged. This research was supported in part by PL-Grid Infrastructure.

\section{LITERATURE}

[1] NAWAZ M., SHAKOOR R.A., KAHRAMAN R., MONTEMOR M.F., 2021, Cerium Oxide Loaded with Gum Arabic As Environmentally Friendly Anti-Corrosion Additive for Protection of Coated Steel, Materials and Design, 198, 109361, https://doi.org/10.1016/j.matdes.2020.109361.

[2] DE SOUZA J.F.T., PACCA S.A., 2021, Carbon Reduction Potential and Costs Through Circular Bioeconomy in the Brazilian Steel Industry, Resources, Conservation and Recycling, 169, https://doi.org/10.1016/j.resconrec. 2021.105517.

[3] GHANAVATI R., NAFFAKH-MOOSAVY H., 2021, Additive Manufacturing of Functionally Graded Metallic Materials: A Review of Experimental and Numerical Studies, Journal of Materials Research and Technology, 13, 1628-1664, https://doi.org/10.1016/j.jmrt.2021.05.022.

[4] ZHANG C., OUYANG D., PAULY S., LIU L., 2021, 3D Printing of Bulk Metallic Glasses, Materials Science and Engineering, R: Reports, 145, 100625, https://doi.org/10.1016/j.mser.2021.100625.

[5] DELKOWSKI M., SMITH C.T.G., ANGUITA V.J., SILVA S.R.P., 2021, Increasing the Robustness and Crack Resistivity of High-Performance Carbon Fiber Composites for Space Applications, IScience, 24, https://doi.org/ 10.1016/j.isci.2021.102692.

[6] MAY M., RUPAKULA G.D., MATURA P., 2020, Non-Polymer-Matrix Composite Materials for Space Applications, Composites Part C: Open Access, 3, 100057, https://doi.org/10.1016/j.jcomc.2020.100057.

[7] FONSTEIN N., 2015, Advanced High Strength Sheet Steels, 1st ed., Springer International Publishing, https://doi. org/ 10.1007/978-3-319-19165-2.

[8] LAZURENKO D.V., LOZANOV V.V., STARK A., PYCZAK F., RUKTUEV A.A., EMURLAEV K.I., SONG L., BATAEV I.A., IVANOV I.V., OGNEVA T.S., BATAEV A.A., 2021, In Situ Synchrotron X-Ray Diffraction Study of Reaction Routes in Ti-Al3Ti-Based Composites: The Effect of Transition Metals on L12 Structure Stabilization, Journal of Alloys and Compounds, 875, 160004, https://doi.org/10.1016/j.jallcom.2021.160004.

[9] DOU K., LORDAN E., ZHANG Y.J., JACOT A., FAN Z.Y., 2020, A Complete Computer Aided Engineering (CAE) Modelling and Optimization of High Pressure Die Casting (HPDC) Process, Journal of Manufacturing Processes, 60, 435-446, https://doi.org/10.1016/j.jmapro.2020.10.062.

[10] MADEJ L., 2017, Digital/Virtual Microstructures in Application to Metals Engineering - A Review, Archives of Civil and Mechanical Engineering, 17, 839-854, https://doi.org/10.1016/j.acme.2017.03.002.

[11] ZHANG X., GUO X., SONG K., WANG X., FENG J., LI S., LIN H., 2021, Simulation and Verification of Thermal Conductivity of CuCr30 Contact Material Based on Morphological Changes of Cr Particles, Materials Today Communications, 26, 102153, https://doi.org/10.1016/j.mtcomm.2021.102153.

[12] BARGMANN S., KLUSEMANN B., MARKMANN J., SCHNABEL J.E., SCHNEIDER K., SOYARSLAN C., WILMERS J., 2018, Generation of 3D Representative Volume Elements for Heterogeneous Materials: A Review, Progress in Materials Science, 96, 322-384, https://doi.org/https://doi.org/10.1016/j.pmatsci.2018.02.003. 
[13] MIN K.M., JEONG W., HONG S.H., LEE C.A., CHA P.R., HAN H.N., LEE M.G., 2020, Integrated Crystal Plasticity and Phase Field Model for Prediction of Recrystallization Texture and Anisotropic Mechanical Properties of Cold-Rolled Ultra-Low Carbon Steels, International Journal of Plasticity, 127, 102644, https://doi.org/10.1016/j.ijplas.2019.102644.

[14] SZELIGA D., BZOWSKI K., RAUCH Ł., KUZIAK R., PIETRZYK M., 2020, Mean Field and Full Field Modelling of Microstructure Evolution and Phase Transformations During Hot Forming and Cooling of Low Carbon Steels, Computer Methods in Material Science, 20, 121-132, https://doi.org/10.7494/cmms.2020.3.0727.

[15] CHEN K., LI H., JIANG Z., LIU F., KANG C., MA X., ZHAO B., 2021, Multiphase Microstructure Formation and Its Effect on Fracture Behavior of Medium Carbon High Silicon High Strength Steel, Journal of Materials Science and Technology, 72, 81-92, https://doi.org/10.1016/j.jmst.2020.09.034.

[16] SONG C., WANG H., SUN Z., WEI Z., YU H., CHEN H., WANG Y., LU J., 2020, Effect of Multiphase Microstructure on Fatigue Crack Propagation Behavior in TRIP-Assisted Steels, International Journal of Fatigue, 133, 105425, https://doi.org/10.1016/j.ijfatigue.2019.105425.

[17] MADEJ L., RAUCH L., PERZYNSKI K., CYBULKA P., 2011, Digital Material Representation As an Efficient Tool for Strain Inhomogeneities Analysis at the Micro scale Level, Archives of Civil and Mechanical Engineering, 11, 661-679, https://doi.org/10.1016/S1644-9665(12)60108-3.

[18] SZYNDLER J., MADEJ Ł., 2015, Numerical Analysis of the Influence of Number of Grains, FE Mesh Density and Friction Coefficient on Representativeness Aspects of the Polycrystalline Digital Material Representation Plane Strain Deformation Case Study, Computational Materials Science, 96, 200-213, https://doi.org/10.1016/ j.commatsci.2014.08.037.

[19] LEWIS A.C., GELTMACHER A.B., 2006, Image-Based Modeling of the Response of Experimental 3D Microstructures to Mechanical Loading, Scripta Materialia, 55, 81-85, https://doi.org/10.1016/j.scriptamat. 2006.01.043.

[20] SZYNDLER J., MADEJ L., 2015, Comparison of 3D and 2D Digital Material Representation Channel Die Compression Results, Materials Science \& Technology, Columbus, 619-626.

[21] BOGUŃ K., SITKO M., MOJŻESZKO M., MADEJ Ł., 2021, Cellular Automata-Based Computational Library for Development of Digital Material Representation Models of Heterogenous Microstructures, Archives of Civil and Mechanical Engineering, 21, 61, https://doi.org/10.1007/s43452-021-00211-9.

[22] SITKO M., MOJZESZKO M., RYCHLOWSKI L., CIOS G., BALA P., MUSZKA K., MADEJ L., 2020, Numerical Procedure of Three-Dimensional Reconstruction of Ferrite-Pearlite Microstructure Data from SEM/EBSD Serial Sectioning, Procedia Manufacturing, 47, 1217-1222 https://doi.org/10.1016/j.promfg.20 20.04.183.

[23] ZANKEL A., WAGNER J., POELT P., 2014, Serial Sectioning Methods for 3D Investigations in Materials Science, Micron, 62, 66-78, https://doi.org/10.1016/j.micron.2014.03.002.

[24] POKHAREL R., LIND J., LI S.F., KENESEI P., LEBENSOHN R.A., SUTER R.M., ROLLETT A.D., 2015, InSitu Observation of Bulk 3D Grain Evolution During Plastic Deformation in Polycrystalline Cu, International Journal of Plasticity, 67, 217-234, https://doi.org/10.1016/j.ijplas.2014.10.013.

[25] DI CAPRIO D., STAFIEJ J., LUCIANO G., ARURAULT L., 2016, 3 D Cellular Automata Simulations of Intra and Intergranular corrosion, Corrosion Science, 112, 438-450, https://doi.org/10.1016/j.corsci.2016.07.028.

[26] HAJDER L., MADEJ L., 2020, Sphere Packing Algorithm for the Generation of Digital Models of Polycrystalline Microstructures with Heterogeneous Grain Sizes, Computer Methods in Materials Science, 20, 22-30.

[27] MAAZI N., LEZZAR B., 2020, An Efficient Monte Carlo Potts Method for the Grain Growth Simulation of SinglePhase Systems, Computer Methods in Material Science, 20, 85-94, https://doi.org/10.7494/cmms.2020.3.0722.

[28] XUE Y., TAKATA N., LI H., KOBASHI M., YUAN L., 2021, Critical Resolved Shear Stress of Activated Slips Measured by Micropillar Compression Tests for Single-Crystals of Cr-Based Laves Phases, Materials Science and Engineering A, 806, 140861, https://doi.org/10.1016/j.msea.2021.140861.

[29] VEENADEVI S.V., ANANTH A.G., 2012, Fractal Image Compression Using Quadtree Decomposition and Huffman Coding, Signal \& Image Processing An International Journal, 3, 207-212, https://doi.org/10.5121/sipij. 2012.3215.

[30] SLOAN S.W., HOULSBY G.T., 1984, An Implementation of Watson's Algorithm for Computing 2-Dimensional Delaunay Triangulations, Advances in Engineering Software, 6/4, 192-197, https://doi.org/10.1016/0141-1195 (84)90003-2.

[31] FABRI A., 1988, Voronoi Diagrams in CGAL, the Computational Geometry Algorithms Library, Communications in Applied Numerical Methods, 4, 709-712, https://doi.org/10.1109/ISVD.2007.46.

[32] LIN T.J., GUAN Z.Q., CHANG J.H., LO S.H., 2014, Vertex-Ball Spring Smoothing: An Efficient Method for Unstructured Dynamic Hybrid Meshes, Computers and Structures, 136, 24-33, https://doi.org/10.1016/j.comp struc.2014.01.028. 
[33] MADEJ L., SITKO M., RADWANSKI K., KUZIAK R., 2016, Validation and Predictions of Coupled Finite Element and Cellular Automata Model: Influence of the Degree of Deformation on Static Recrystallization Kinetics Case Study, Materials Chemistry and Physics, 179, 282-294, https://doi.org/10.1016/j.matchemphys. 2016.05.040.

[34] SITKO M., CHAO Q., WANG J., PERZYNSKI K., MUSZKA K., MADEJ L., 2020, A Parallel Version of the Cellular Automata Static Recrystallization Model Dedicated for High Performance Computing Platforms - Development and Verification, Computational Materials Science, 172, 109283, https://doi.org/10.1016/j.com matsci.2019.109283. 